\title{
Hybrid-Reanimations-Schulungen (Basic-Life-Support) - ein Modell für die Zukunft?
}

Eine rasche Reanimation ist in Akutsituationen überlebenswichtig. Das Training dieser Fähigkeiten und Fertigkeiten bildet die Grundlage für einen reibungslosen und erfolgreichen Einsatz im Notfall. Daher werden im Universitätsklinikum St. Pölten alle Mitarbeiterinnen und Mitarbeiter auf lebensrettende Sofortmaßnahmen geschult. Im Rahmen der COVID-19-Pandemie konnte durch den Einsatz einer neuen hybriden Schulungsvariante eine Möglichkeit geschaffen werden, den Mitarbeiterinnen und Mitarbeitern Sicherheit und Routine bei der Durchführung dieser Sofortmaßnahmen zu geben.

\section{Markus Korntheurer,}

Patrick Pernerstorfer

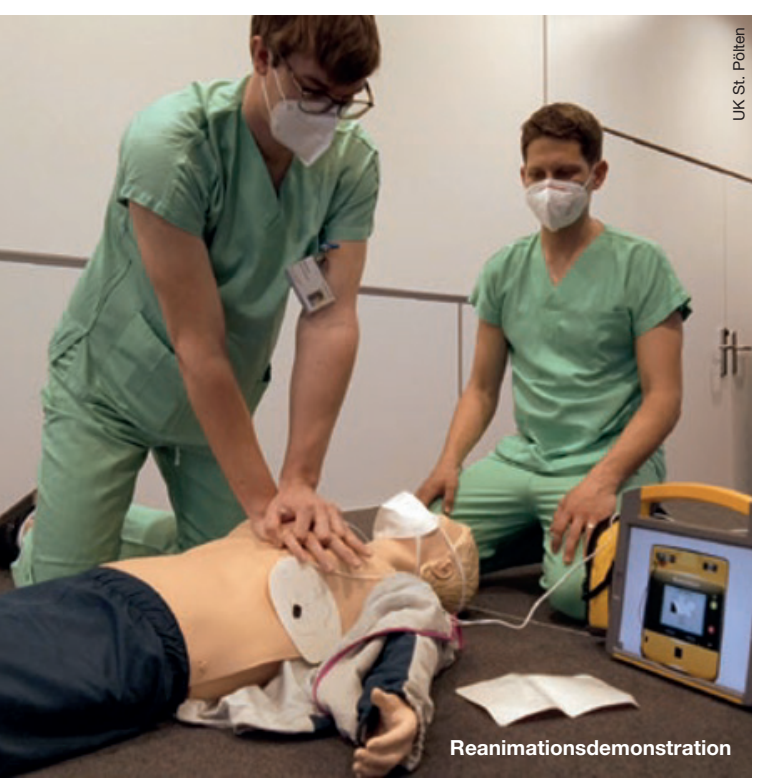

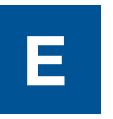
ntwickelt wurde diese hybride Variante des Reanimationstrainings von zwei diplomierten Gesundheits- und Krankenpflegern, die eine alternative Möglichkeit für die gesetzlich verpflichtende Fortbildung in Zeiten der Pandemie erfolgreich umgesetzt haben. Die theoretischen Grundlagen werden mithilfe eines Skriptums sowie eines Videotutorials vermittelt. Für das praktische Training werden in Kleingruppen die notwendigen Handgriffe und Abläufe in Fallsimulationen mithilfe einer Reanimationspuppe sowie eines Übungsmonitors geübt.

Die Covid-19-Pandemie hat uns in den letzten 1,5 Jahren gezwungen, in vielen Bereichen des Lebens umzudenken, aber gleichsam auch die Chance gegeben, bisher gelebte und bekannte Strukturen zu evaluieren und zu adaptieren. Einer dieser Bereiche ist für das Universitätsklinikum St. Pölten die innerklinischen Fortbildungen im Bereich Notfallmanagement und Reanimation. Durch die hohe Arbeitsbelastung in Zeiten der Pandemie und die Vorgaben, alle Infektionsmöglichkeiten zu reduzieren, konnten Trainings nur punktuell und eingeschränkt stattfinden.

Durch die Durchführung von regelmäßigen Reanimationsschulungen inkl. Trainings kann das Verhalten in Notfallsituationen simuliert werden. Durch diese Simulationsübungen kommt es bei den Mitarbeiterinnen und Mitarbeitern zu einer Festigung des Wissens und der Fertigkeiten sowie zu einem sicheren Umgang im Notfallmanagement. Nur durch dieses fachgerechte
Handling kann der Outcome für die Patientinnen und Patienten gesteigert werden. Fokus liegt hier ebenso auf der Reduzierung der potenziellen Infektionsgefahr durch fachgerechtes Handling.

Um derzeit trotz der Pandemie den Wissensstand der Mitarbeiterinnen und Mitarbeiter durch regelmäßige Schulungen sicherstellen zu können und auch in eventuellen weiteren Phasen des Lockdowns ein Konzept für die Reanimationsschulungen zu haben, wurde seitens der Pflegedirektion in Kooperation mit zwei Angehörigen des gehobenen Dienstes für Gesundheits- und Krankenpflege ein Schulungskonzept für innerklinische Reanimationsschulungen ausgearbeitet.

\section{Ausgangslage/bisheriges Schulungskonzept}

Das bisherige Schulungskonzept für den Pflegebereich sah eine Schulungsmaßnahme von vier Stunden Präsenzveranstaltung vor. Diese fand einmal im Monat statt und war für nach interner Anmeldung (begrenzte Teilnehmerzahl) frei zugänglich. Das Konzept setzte auf Wissenstransfer in Form von Präsenzveranstaltungen. Dieses Konzept war im Rahmen der Pandemie durch die Mitarbeiterbindung über mehrere Stunden hinweg und das Zusammentreffen von mehreren Personen aus verschiedenen Abteilungen nicht weiter aufrecht zu erhalten. Zur Sicherstellung der Reanimationskompetenz in Zeiten von COVID-19 war hier ein innovativer Lösungsansatz gefordert. 


\section{Zielsetzung}

Das Ziel war es, ein innovatives Konzept zu entwickeln, das auch über die Krise hinaus von großem Nutzen sein kann. Die Idee war, den Mitarbeitern ein mehrstufiges Konzept mit semiflexibler Durchführung anbieten zu können, das auch in Zeiten der Pandemie durchführbar ist. Das Konzept bietet einen Theorie- sowie Praxisteil, welche zeitlich getrennt durchgeführt werden können. Der Theoriepart gliedert sich in ein Video, das ein Notfallszenario im klinischen Bereich simuliert, und das Selbststudium eines Kursskriptums, das den theoretischen Input realitätsnah vermittelt. Eine eigenständige Wissensüberprüfung rundet den theoretischen Part ab. Zum Abschluss dieser Wissensüberprüfung erhalten die Teilnehmer eine Teilnahmebestätigung, die wiederum als Zulassung zum praktischen Kleingruppentraining zu sehen ist.

Durch den theoretischen Teil soll eine gute Basis für die darauffolgenden Kleingruppentrainings geschaffen werden, in denen bis zu vier Teilnehmer die theoretischen Inhalte mittels eines Simulationstrainings mit Unterstützung eines Instruktors in die Praxis umsetzen.

Dies führte zu einer raschen Umsetzungsmöglichkeit und inkludiert eine flexible Zeiteinteilung bei der eigenständigen $\mathrm{Ab}$ solvierung des theoretischen Teils. Die Mitarbeiter haben die Möglichkeit, bei Bedarf immer wieder die theoretischen Grundlagen (Video und Schulungsunterlagen) an- bzw. nachzusehen. Dieses Konzept ermöglicht somit einen stetigen Zugriff auf aktuelles Wissen zum Thema Reanimation sowie die Chance, eine verpflichtende Trainingseinheit gewährleisten zu können. Durch die stetige Schulung soll den Mitarbeitern die Sicherheit im Handling mit Notfallsituationen gegeben werden.

\section{Grundlagen}

Grundlage für den theoretischen Teil des Reanimationstrainings sind die Empfeh- lungen der ARC (Österreichischer Rat für Wiederbelebung) (Schwindt et.al, 2020).

Das Vermittlungskonzept basiert auf dem 4-Steps-Approach nach Peyton. Bei dieser Methode werden im ersten Schritt die Fertigkeiten in normaler Geschwindigkeit ohne Kommentare demonstriert. Im Anschluss daran werden die Fertigkeiten in einzelne kleine kommentierte Teilschritte dekonstruiert, bevor im dritten Schritt die Teilnehmer den Vortragenden verbal bei der Tätigkeit anleiten. Abschließend wird die Performance langsam von den Teilnehmern durchgeführt, wobei hier die eigene Kommentierung ebenso beinhaltet ist (Pierre, Breuer, 2013, S. 75).

\section{Projektablauf/Methode}

\section{- Ideenfindung}

- Besprechung mit der Stabsstelle für Personalentwicklung (Pflegedirektion): Abklärung der Rahmenbedingungen bzw. der benötigten Ressourcen

- Video: Planung der Abläufe mit Fokus auf die ersten 2 Schritte nach Peyton, Videodreh und Freigabe durch Beauftragende

- Ausbildungsunterlagen: Erstellung eines Skripts, Rahmenbedingungen für die Durchführung des Theorieteils

- praktische Schulung: Erstellung der SOP (Hygienerichtlinien, Teilnehmeranzahl, Zielgruppenbestimmung), Durchführung der Schritte 3 und 4 nach Peyton

- Kommunikation der hybriden Schulungsmaßnahmen: Pressetext, hausinterne Information, Freischaltung für die Mitarbeiter

- Qualitätsüberprüfung: Feedbackbogen für die Teilnehmerinnen und Teilnehmer (Zufriedenheit, Lerneffekt) zum kontinuierlichen Assessment der Trainings

\section{Qualitätsüberprüfung der Hybridmethode}

Für die Mitarbeiter wurde so die Grundlage geschaffen, jederzeit die theoretischen Inhalte aufzufrischen sowie regelmäßig an den praktischen Reanimationstrainings teilnehmen zu können. Die theoretischen Grundlagen werden mittels Online-Wissensüberprüfung abgefragt und können jederzeit bei Bedarf adaptiert werden. Im praktischen Part werden immer wieder Verständnisfragen gestellt, um so einen möglichst hohen Outcome für die Teilnehmer zu haben. Durch die daraus entstandene Zeitersparnis kann mehreren Mitarbeitern die praktische Schulungsmöglichkeit angeboten werden. Ebenso können dadurch die Schulungsunterlagen bei Bedarf adaptiert werden.

Durch das Feedback per Onlinefragebogen (per QR-Code auf der Teilnahmebestätigung durchführbar) werden die Zufriedenheit und der Lernerfolg erfragt.

Im Mai 2021 konnte erfolgreich mit den ersten Trainings gestartet werden.

Literatur

- Schwindt, J., Pflanzl-Knizacek, L., Nierscher, F.-J., Hoffmann, P., Singraber, E.-M., Cardona, F., Burda, G., Köstenberger, M., Egger, A., Schlieber, J., Baubin, M. (2020): Positionspapier des Österreichischen Rates für Wiederbelebung (Austrian Resuscitation Council - ARC) zur Durchführung von Kursen zur Aus- und Fortbildung von medizinischem Personal in lebensrettenden Maßnahmen während der COVID-19-Pandemie. Österreichischer Rat für Wiederbelebung (ARC). Version 2.0 Zugriff am 25.5.2021 unter Positionspapier_ARC V2.pdf

- St. Pierre, M., Breuer, G. (Hrsg.) - (2013): Simulation in der Medizin: Grundlegende Konzepte - Klinische Anwendung. Springer Verlag

- Bullock lan, Davis Mike, Lockey Andrew, MackwayJones Kevin (2016): Pocket Guide to Teaching for Clinical Instructors. Third Edition. New Jersey: WILEY Blackwell (Methodik)

Autoren:

\section{Markus Korntheurer}

Diplomierter Gesundheits- und

Krankenpfleger, Sonderausbildung

Intensivpflege, ALS Instruktor

Patrick Pernerstorfer

Diplomierter Gesundheits- und

Krankenpfleger, Sonderausbildung

Anästhesie- und Intensivpflege

Kontakt-Email:

pd@stpoelten.Iknoe.at 